\title{
ANALYSIS AND DESIGN OF REINFORCED CONCRETE STRUCTURES AS A TOPOLOGY OPTIMIZATION PROBLEM
}

\author{
M. Bruggi \\ Department of Civil and Environmental Engineering DICA \\ Politecnico di Milano \\ Piazza Leonardo da Vinci, 3220133 Milano Italy \\ e-mail: matteo.bruggi@polimi.it
}

Keywords: Topology Optimization, No-Tension Materials, Reinforced-Concrete Structures, Size Optimization.

\begin{abstract}
Technical codes for buildings deal with cracked reinforced concrete structures assuming concrete as a compression-only material, whereas rebar provides the structural component with the required tensile strength [1]. Numerical methods can handle reinforced concrete structures calling for demanding non-linear analysis. Indeed, well-known convergence issues arise when copying with concrete as a compression-only material. Recently, an alternative energy-based approach has been proposed to solve the equilibrium of a linear elastic notension medium exploiting its hyper-elasticity [2]. A topology optimization problem distributes an equivalent orthotropic material to minimize the strain energy of the no-tension body, thus avoiding more demanding non-linear analysis. This contribution provides an extension to the analysis and optimal design of reinforced concrete structures. Following [3], truss members are modeled within a two-dimensional no-tension continuum in order to model structural elements made of reinforced concrete. The solution of the equilibrium is straightforward within the approach proposed in [2], thus allowing performing analysis at the serviceability limit state with cracked sections. Also, introducing the areas of the reinforcement bars as an additional set of unknowns, a problem of size optimization is outlined to cope with the optimal rebar of r.c. structures. Preliminary numerical simulations are shown to assess the proposed procedure.
\end{abstract}




\section{INTRODUCTION}

An extensive research has been done in the last decades addressing the non-linear analysis of reinforced concrete (r.c.) structures, see e.g. the seminal paper [4]. At the serviceability limit state, the practical detailing of a reinforced concrete structure is based on simple assumptions, i.e. the adoption of linear elastic modeling for the composite structural member and the assumption that concrete strength in tension is negligible [5]. Implementing this theoretical model within a numerical code is not trivial, due to well-known instabilities arising when addressing a linear elastic no-tension body through conventional incremental approaches. Recently, [6] has proposed to solve the equilibrium of a no-tension solid resorting to a minimization problem that adopts the displacement field as unknown and the strain energy of the hyper-elastic no-tension body as objective function.

An alternative energy-based approach has been proposed in [2]. A topology optimization problem [7] distributes an equivalent orthotropic material to minimize the strain energy of the compression-only body, thus resorting to an established and computationally efficient formulation of compliance minimization that avoids more demanding non-linear analysis.

A possible extension to the simplified analysis of reinforced concrete structures is investigated in this contribution. Following [3], truss members are modeled within a two-dimensional no-tension continuum in order to address two-dimensional structural elements made of reinforced concrete. The solution of the equilibrium is straightforward within the approach proposed in [2], thus allowing performing analysis at the serviceability limit state with cracked sections. Preliminary numerical simulations are shown to investigate the capabilities of the proposed procedure.

Moreover, introducing the areas of the reinforcement bars as an additional set of unknowns for the minimization problem, size optimization is outlined as a possible extension of the proposed procedure to cope with the optimal rebar of r.c. structures. A first numerical test shows that the computational burden tied to this extension is almost equivalent to that required for the solution of the equilibrium equations.

The outline of the paper is as follows. Section 2.1 recalls fundamentals of the topology optimization problem used to address linear elastic no-tension structures according to [2, 8], whereas Section 2.2 introduces the minimum compliance problem to cope with r.c. members. Section 3 presents preliminary numerical simulations performed on a benchmark example and Section 4 provides remarks and outlines the ongoing research.

\section{PROBLEM FORMULATION}

\subsection{Equilibrium of no-tension structures as a topology optimization problem}

The analysis of a 2D no-tension continuum is re-formulated as a topology optimization problem for minimum compliance. The equilibrium of any compression-only structure is solved seeking for the distribution of an 'equivalent' orthotropic material that minimizes the potential energy of the hyper-elastic solid. A one-shot energy-based procedure computes the non-incremental solution under given loads, provided that the applied forces are compatible with the no-tension constraint.

A 2D Cartesian reference is considered to address a no-tension isotropic solid in the region $\Omega$. Prescribed displacements $\underline{u}_{0}$ are enforced along its constrained boundary, $\Gamma_{u}$, whereas traction $\underline{t}_{0}$ is assigned along its free boundary, $\Gamma_{t}$. An equivalent orthotropic material is introduced to mimic the behavior of a no-tension body. $\left(\widetilde{z}_{1}, \widetilde{z}_{2}\right)$ are the symmetry axes of the equivalent phase that are assumed to be aligned with the directions $\left(z_{I}, z_{I I}\right)$ of the principal stresses 
$\left(\sigma_{I}, \sigma_{I I}\right)$ at any point in $\Omega$. The parameter $\theta$ defines the orientation of the axes $\left(z_{I}, z_{I I}\right)$ with respect to the given Cartesian reference.

In weak form, the energy-based minimization problem can be stated as follows:

$$
\begin{cases}\min _{\rho_{\min } \leq \rho_{1}, \rho_{2} \leq 1} & \int_{\Omega} \mathbf{D}\left(\rho_{1}, \rho_{2}, \theta\right) \underline{\varepsilon}(\underline{u}) \underline{\varepsilon}(\underline{u}) d \Omega \\ \text { s.t. } & \int_{\Omega} \mathbf{D}\left(\rho_{1}, \rho_{2}, \theta\right) \underline{\varepsilon}(\underline{u}) \underline{\varepsilon}(\underline{v}) d \Omega=\int_{\Gamma_{t}} \underline{t}_{0} \cdot \underline{v} d \Gamma \text { and }\left.\underline{u}\right|_{\Gamma_{u}}=\underline{u}_{0} \quad \forall \underline{v}, \\ & \theta \mid \widetilde{z}_{1}=z_{I} \text { and } \widetilde{z}_{2}=z_{I I}, \\ & \rho_{1}, \rho_{2} \mid \sigma_{I} \leq 0 \text { and } \sigma_{I I} \leq 0 .\end{cases}
$$

The above equation adopts the compliance (i.e. twice the strain-energy) as objective function, $\underline{u}$ is the displacement field, $\underline{\varepsilon}(\underline{u})$ the strain tensor and $\mathbf{D}$ the fourth-order elasticity tensor of the 'equivalent' orthotropic material. Using a suitable transformation matrix $\mathbf{T}(\theta)$, one has that $\mathbf{D}=\mathbf{T}^{-1} \widetilde{\mathbf{D}} \mathbf{T}^{-t}$. Exploiting the Voigt's notation, $\widetilde{\mathbf{D}}$, which is the constitutive tensor written in the material frame $\left(\widetilde{z}_{1}, \widetilde{z}_{2}\right)$, reads:

$$
\widetilde{\mathbf{D}}=\frac{1}{1-\widetilde{\nu}_{12} \widetilde{\nu}_{21}}\left[\begin{array}{ccc}
\widetilde{E}_{1} & \widetilde{\nu}_{12} \widetilde{E}_{2} & 0 \\
\widetilde{\nu}_{21} \widetilde{E}_{1} & \widetilde{E}_{2} & 0 \\
0 & 0 & \left(1-\widetilde{\nu}_{12} \widetilde{\nu}_{21}\right) \widetilde{G}_{12}
\end{array}\right]
$$

where $\widetilde{E}_{1}$ and $\widetilde{E}_{2}$ are the Young's moduli of the 'equivalent' orthotropic material, $\widetilde{\nu}_{12}, \widetilde{\nu}_{21}$ its Poisson's ratios (being $\widetilde{\nu}_{12} / \widetilde{E}_{1}=\widetilde{\nu}_{21} / \widetilde{E}_{2}$ ) and $\widetilde{G}_{12}$ its shear modulus.

A generalization of the so-called SIMP model [9] relates the elastic constants of the equivalent material and those $(E, \nu)$ of the isotropic no-tension material according to the following interpolations:

$$
\widetilde{E}_{i}=\rho_{i}^{p} E, \quad \widetilde{\nu}_{i j}=\sqrt{\frac{\rho_{i}^{p}}{\rho_{j}^{p}}} \nu, \quad \widetilde{G}_{i j}=\sqrt{\rho_{i}^{p} \rho_{j}^{p}} \frac{E}{2(1+\nu)},
$$

for $i, j=1,2$. Each minimization unknown $\rho_{i}$ ranges between $\rho_{\min }>0$ and 1 to penalize or preserve stiffness along the relevant axis depending on the sign of the principal stress. To avoid any tensile stress in the solid, Eqns. (1,4) enforce vanishing stiffness of the equivalent orthotropic material along the direction of any arising positive principal stress. The adopted lower bound $\rho_{\min }$ avoids singularity of the stiffness matrix when solving the discrete formulation, whereas $p=3$ is conventionally assumed [7].

\subsection{Equilibrium of r.c. structures as a topology optimization problem}

The generalization of the above problem to the finite element analysis of a cracked reinforced concrete structure seen as a strengthened no-tension body is straightforward. Following [3], truss elements are modeled within a two-dimensional continuum in order to cope with the arising composite structure. The formulation is presented in its discrete form.

A finite element discretization made of $N$ truss elements is used for the reinforcement bars, along with $M$ four-node plane elements for the underlying compression-only structure. An element-wise constant discretization is adopted to cope with $\rho_{1}, \rho_{2}, \theta$, see Section 2.1. The stiffness matrix of the $j$-th truss-like element is denoted by $\mathbf{K}_{j}^{r}$, whereas $x_{1 i}$ and $x_{2 i}$ are the discrete minimization unknowns that govern the stiffness of the 'equivalent material' along its symmetry axes, being $t_{i}$ the value of the orientation parameter in the $i$-th element. Denoting by 
$\mathbf{K}_{i}\left(x_{1 i}, x_{2 i}, t_{i}\right)$ the stiffness matrix of the $i$-th plane element, the implemented discrete form for the minimization of the strain energy of a $2 \mathrm{D}$ r.c. structure reads:

$$
\begin{cases}\min _{x_{\min } \leq x_{1 i}, x_{2 i} \leq 1} & \mathcal{C}=\sum_{i=1}^{M} \mathbf{U}_{i}^{T} \mathbf{K}_{i}\left(x_{1 i}, x_{2 i}, t_{i}\right) \mathbf{U}_{i}+\sum_{j=1}^{N} \mathbf{U}_{j}^{T} \mathbf{K}_{j}^{r} \mathbf{U}_{j} \\ \text { s.t. } & \sum_{i=1}^{M} \mathbf{K}_{i}\left(x_{1 i}, x_{2 i}, t_{i}\right) \mathbf{U}+\sum_{j=1}^{N} \mathbf{K}_{j}^{r} \mathbf{U}_{j}=\mathbf{F}, \\ & t_{i} \mid \widetilde{z}_{1}=z_{I} \text { and } \widetilde{z}_{2}=z_{I I}, \\ & x_{1 i}, x_{2 i} \mid \sigma_{i, I} \leq 0 \text { and } \sigma_{i, I I} \leq 0,\end{cases}
$$

where $\mathbf{U}_{i}$ is the vector of the d.o.f.s of the $2 \mathrm{D}$ finite elements, $\mathbf{U}_{j}$ is the vector of the d.o.f.s of the truss elements and $F$ is the array of the nodal loads. Eqn.(4) is fully along the lines of Eqn.(1). It is recalled that the structural compliance $\mathcal{C}$ is the work of the external loads computed at equilibrium. Dealing with a composite structure, the overall strain energy depends on the amount stored in the underlying compression-only material and that stored in the steel reinforcement.

Sequential convex programming [10] and analytical computation of the sensitivities [7] can be used to solve the minimization problem stated in Eqn.(4).

Instead of implementing demanding sets of stress constraints, the penalization approach already used in [2, 8] is herein adopted to cope with Eqn.(4,4). A set of penalized densities $\widehat{x}_{1 i}$, $\widehat{x}_{2 i}$ can be introduced for a straightforward computation of a modified strain energy $\widehat{\mathcal{C}}$, in which the terms related to any possible positive principal stress arising in the underlying compressiononly material are reduced by a parameter $k=0.5$. Providing the optimizer with the reduced objective function $\widehat{\mathcal{C}}$ and its sensitivities $\partial \widehat{C} / \partial x_{1 i}, \partial \widehat{\mathcal{C}} / \partial x_{2 i}$, variables $\mathbf{x}_{1}, \mathbf{x}_{2}$ are updated preventing any distribution of stiff material along the weak direction(s) of the no-tension body.

Introducing a new set of minimization unknowns to cope with bars having different sections, minor modifications are required in Eqn.(4) to cope with a problem of size optimization. A preview of this approach is shown in Section 3.2, addressing the optimal detailing of r.c. structures.

\section{NUMERICAL SIMULATIONS}

A set of preliminary numerical simulations are presented in this section, adopting the formulation described above to cope with the analysis of the reinforced concrete cantilever represented in Figure 1, The left edge is clamped and a nodal force $P=1 \mathrm{kN}$ is applied at the upper right corner.

Concrete is modeled as a linear elastic no-tension material with Young modulus $E_{c}=$ $20,000 \mathrm{MPa}$ and Poisson's ratio $\nu_{c}=0.15$, whereas the prescribed steel reinforcement is discretized through linear elastic truss elements with Young modulus $E_{s}=210,000 \mathrm{MPa}$. The



Figure 1: Geometry and boundary conditions for the numerical application. 




Figure 2: Finite element mesh and steel reinforcement layout.

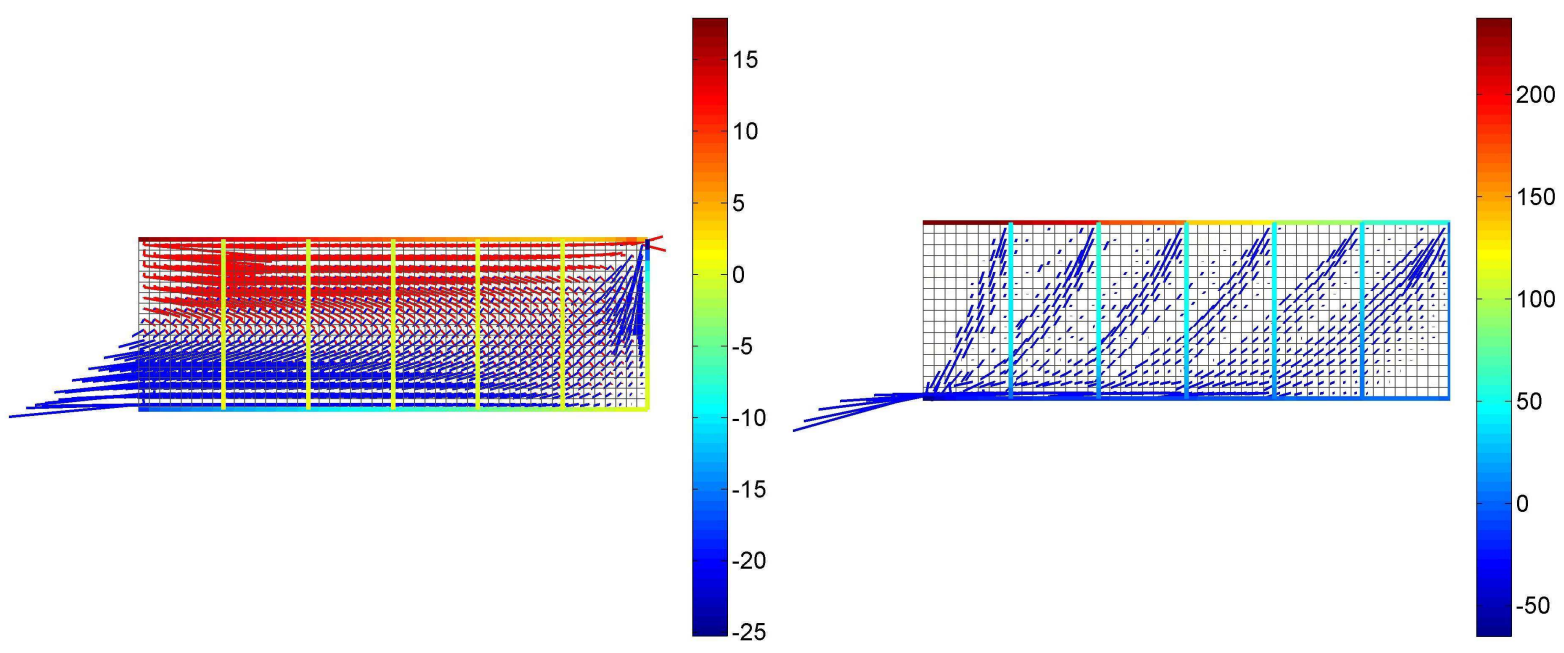

(a)

(b)

Figure 3: Analysis of the r.c. cantilever: principal stresses in the concrete specimen along with uniaxial stresses in the reinforcement (MPa) computed for a conventional linear elastic beam (a) and a no-tension beam (b).

area of each bar is twice that of a circular section with diameter $\phi_{s}=12 \mathrm{~mm}$.

Section 3.1 assesses the proposed procedure of analysis, whereas Section 3.2 outlines a possible extension to the size optimization of the steel reinforcement.

\subsection{The analysis problem}

Figure 2 shows the two-dimensional finite element discretization used to handle the concrete cantilever along with the truss elements that model stirrups and longitudinal reinforcement, both with the same diameter $\phi_{s}$. For simplicity's sake, no reinforcing covering is considered in this preliminary investigation. Stirrup spacing is uniform along the beam and equal to half the height of the square section.

Figure 3(a) shows results computed through a linear elastic analysis that models concrete as a material with equal behavior in tension and compression. Principal stresses in the concrete specimen and uniaxial stresses in the reinforcement are represented in the same picture. Due to the enforced compatibility, the steel reinforcement and the concrete beam carry different amounts of the external load, depending on their stiffness. As expected, the upper part of the beam is tensile-stressed (red vectors), whereas the region under the neutral axis is compressed (blue vectors). Minor axial stresses are found in the longitudinal reinforcement; stirrups are nearly unloaded. 


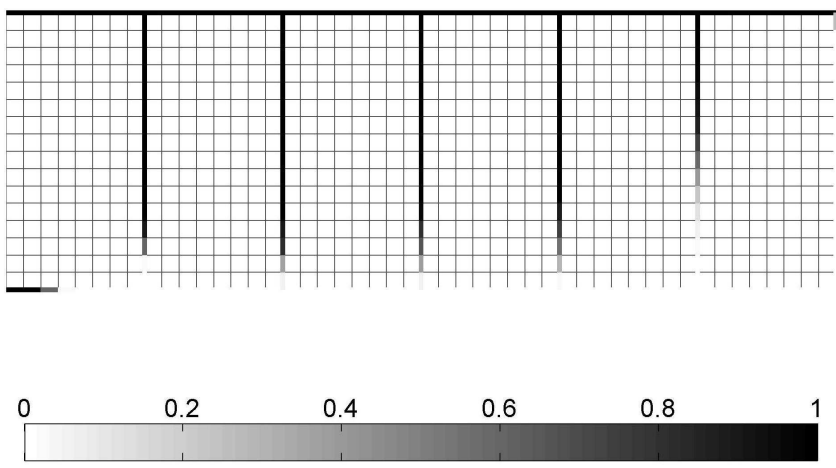

Figure 4: Optimization of the r.c. cantilever: achieved layout.

Figure 3(b) shows results computed through the minimization problem of Eqn. (4), which allows modeling concrete as a linear elastic no-tension material. Results shown in this picture are quite different with respect to those of the previous one. No tensile stress arises in the concrete body (no red vector is found). The tensile stresses in the upper longitudinal reinforcement are around $230 \mathrm{MPa}$, whereas compressive stresses in the lower horizontal bars are around $50 \mathrm{MPa}$.

The direction of the principal stresses computed in the two-dimensional concrete domain clearly shows the expected activation of a strut-and-tie model for shear resistance that involves the stirrups, now tensile-stressed by nearly $50 \mathrm{MPa}$. Note that the inclination of the struts in the arising truss-like structure is not the same along the cantilever. This can be directly compared with established results of strut-and-tie modeling, see e.g. [1, 11].

\subsection{The optimization problem}

The formulation in Eqn. (4) can be straightforwardly modified to cope with the detailing of the optimal amount of steel reinforcement in the cantilever.

Replacing the stiffness of the $j$-th reinforcement bar $\mathbf{K}_{j}^{r}$ with $x_{j} \mathbf{K}_{j}^{r}$, being $0 \leq x_{j} \leq 1$ a sizing unknown that allows for a variation of the diameter of the bar section in the range $0 \leq \phi_{s} \leq 12 \mathrm{~mm}$, the problem in Eqn. (4) minimizes the strain energy of the composite structure solving, within the same formulation, the equilibrium of the no-tension cantilever and the size optimization of the prescribed reinforcement bars.

Figure 4 shows the distribution of the unknowns $x_{j}$ found by the algorithm to detect optimal sections, enforcing that the allowed global amount of reinforcement is half the case with $x_{j}=1, \forall j$. Figure 5 represents the principal compressive stresses found in the concrete specimen along with the uniaxial stresses computed in the reinforcement. Removing the horizontal reinforcement lying in the compressive-stressed region and suitable portions of the stirrups, a lighter layout is achieved than in Figure 2 without introducing any remarkable variation in the computed stress field. The achieved solution is trivial, but allows outlining capabilities of the proposed formulation that can be conveniently exploited in case of more complex geometry, load and reinforcement patterns. 


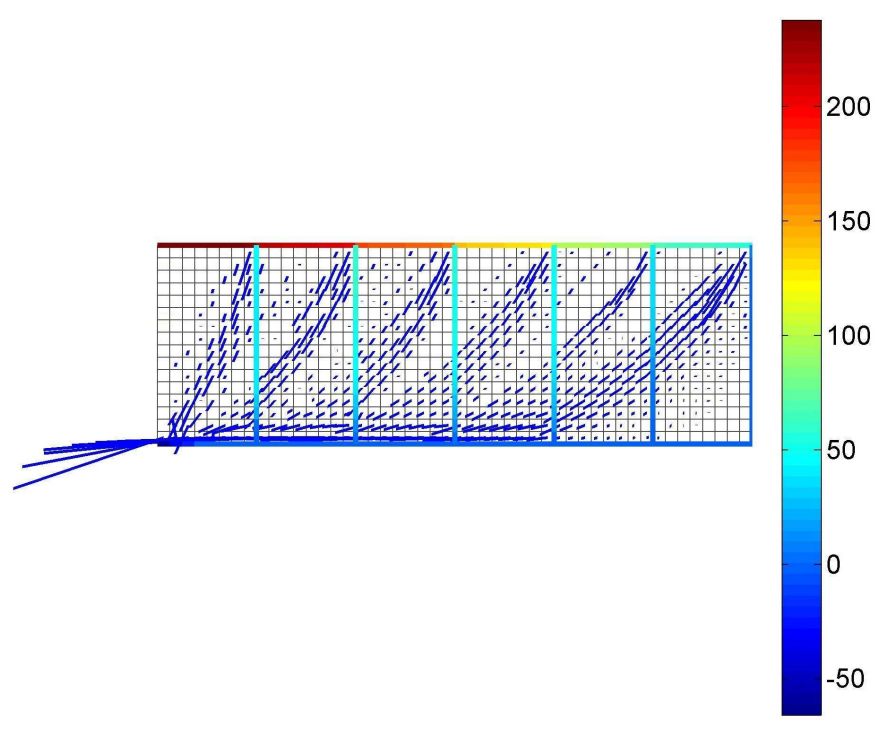

Figure 5: Optimization of the r.c. cantilever: principal stresses in the concrete specimen along with uniaxial stresses in the reinforcement $(\mathrm{MPa})$.

Figure 6 reports the history plot of the objective function for the analysis problem and the optimization problem that have been considered above. Both curves are similar, convergence is smooth and the computational cost is limited, as for conventional problems of topology optimization for minimum compliance.

\section{CONCLUSIONS}

A topology optimization problem has been formulated as an alternative approach to cope with the analysis of cracked reinforced concrete structures, assuming the hyper-elastic notension model for concrete. An energy-based formulation which was originally developed for plain elements has been herein extended to handle compression-only composite structures embedding bars of steel reinforcement.

Results found by the preliminary analysis commented above are in very good agreement with established theories addressing the behavior of cracked reinforced-concrete structures, see e.g. [11]. The proposed approach allows for a direct computation of compressive stresses acting in the concrete domain along with axial stresses in the steel reinforcement. This matches wellknown methods used in engineering practice that neglect the tensile strength of concrete, see e.g. [5].

Moreover, a problem of size optimization has been outlined to cope with the optimal rebar of r.c. sections through minor modifications of the proposed numerical procedure.

The ongoing research is mainly focused on the assessment of the analysis problem, the development of the optimization problem and the extension of the proposed approach to cope with the optimal fiber-reinforcement of existing structures, see in particular [12, 13] for plain and reinforced concrete structures and [14,15] for masonry structures.

Referring to computational issues, adaptive techniques are currently under investigation to improve the accuracy in the evaluation of both the displacement field and the stress field in the no-tension layer while decreasing the computational effort, see in particular [16, 17]. 




Figure 6: Convergence curves for the analysis problem and the optimization problem (Nm).

\section{REFERENCES}

[1] P. Marti, Basic tools of reinforced concrete beam design. Journal of the American Concrete Institute, 82, 46-56, 1985.

[2] M. Bruggi, Finite element analysis of no-tension structures as a topology optimization problem. Structural and Multidisciplinary Optimization, 50(6), 957-973, 2014.

[3] A.L. Gaynor, J.K. Guest, C.D. Moen, Reinforced concrete force visualization and design using bilinear truss-continuum topology optimization. Journal of Structural Engineering, 139, 607-618, 2013.

[4] H.G. Kwak, F.C. Filippou, Nonlinear FE analysis of R/C structures under monotonic loads. Computers and Structures, 65, 1-16, 1997.

[5] EN 1992-1-1, Eurocode 2: Design of concrete structures Part 1-1: General rules and rules for buildings, 2004.

[6] M. Angelillo, L. Cardamone, A. Fortunato, A numerical model for masonry-like structures. Journal of Mechanics of Materials and Structures 5, 583-615, 2010.

[7] M.P. Bendsøe, O. Sigmund, Topology optimization theory, methods and applications, New York, Springer, 2003.

[8] M. Bruggi, A. Taliercio, Analysis of no-tension structures under monotonic loading through an energy-based method. Computers and Structures 159, 14-25, 2015.

[9] M.P. Bendsøe, Optimal shape design as a material distribution problem. Structural Optimization, 1, 193-202, 1989.

[10] K. Svanberg, Method of moving asymptotes - a new method for structural optimization. International Journal for Numerical Methods in Engineering, 24, 193-202, 1987.

[11] J. Schlaich, K. Schaefer, M. Jennewein, Toward a consistent design of structural concrete. PCI Journal, 32(3), 74-150, 1987. 
[12] M. Bruggi, A. Taliercio, Optimal strengthening of concrete plates with unidirectional fiber-reinforcing layers. International Journal of Solids and Structures, 67-68, 311-325, 2015.

[13] J. Cunha, L.P. Chaves, The use of topology optimization in disposing carbon fiber reinforcement for concrete structures. Structural and Multidisciplinary Optimization, 49(6), 1009-1023, 2014.

[14] M. Bruggi, G. Milani, A. Taliercio, Design of the optimal fiber-reinforcement for masonry structures via topology optimization. International Journal of Solids and Structures, 50(13), 2087-2106, 2013.

[15] M. Bruggi, G. Milani, A. Taliercio, Simple topology optimization strategy for the FRP reinforcement of masonry walls in two-way bending. Computers and Structures, 138, 86$101,2014$.

[16] Y. Wang, Z. Kang, Q. He, Adaptive topology optimization with independent error control for separated displacement and density fields. Computers and Structures, 135, 50-61, 2014.

[17] M. Bruggi, M. Verani, A fully adaptive topology optimization algorithm with goaloriented error control. Computers and Structures, 89(15-16), 1481-1493, 2011. 